\title{
Sustained Improvement in Anemia with Low-Dose Recombinant Human Erythropoietin Therapy in a Patient with Hypoplastic Myelodysplastic Syndrome and Chromosomal Abnormalities
}

\author{
Yoshiko Tamai, Hideki TAKami, Rieko NaKahata, Katsutoshi KariYa and Akihiro MunaKata
}

We present a case report of a 55-year-old male patient with hypoplastic myelodysplastic syndrome (MDS, refractory anemia) in which a good response to recombinant human erythropoietin (rhEPO) has been maintained for more than 60 months. There is with no evidence of progression to high risk MDS or acute leukemia, although he was predicted to be a low-responder to rhEPO therapy because of very high serum EPO levels $(5,260 \mathrm{mU} / \mathrm{ml})$, a history of multiple transfusions, chromosomal abnormalities $(47, \mathrm{XY},+8)$ and severe thrombocytopenia. Since he received rhEPO with no adverse effects, it may be valuable to try rhEPO treatment at least one time for low-risk MDS patients, depending on red cell transfusion requirements.

(Internal Medicine 37: 320-323, 1998)

Key words: myelodysplastic syndrome (MDS), erythropoietin, serum erythropoietin concentration, predictive factors

\section{Introduction}

Myelodysplastic syndrome (MDS) is a group of clonal stem cell disorders which to date lack effective treatment. Bone marrow transplantation (BMT) may be an effective treatment for patients under age 40 , however, $70 \%$ of MDS patients are over age 50. Although infections are the most critical lifethreatening episodes, most patients are more concerned with the anemia and subsequent need for repeated red cell transfusions required for normal daily activities. However, repeated transfusions have a high risk of alloimmunization, transmission of viral diseases and iron overload, with a decrease in the quality of life and increase in morbidity. Recently, recombinant human erythropoietin (rhEPO) has become available for clinical use in patients with MDS with erythroid responses occurring in 20 to $34 \%$ of patients $(1,2)$, but fewer patients have achieved transfusion-free state. We report a patient with hypoplastic MDS with chromosomal abnormalities in which a good response to rhEPO has been maintained for more than 60 months without adverse effects.

For editorial comment, see also p 235.
The administration of rhEPO for this case was approved by the committees of the clinical trials and ethics of Hirosaki University School of Medicine, and written informed consent was obtained from the patient.

\section{Case Report}

A 55-year-old male was seen in our hospital in January 1991 with general malaise and nasal bleeding and was found to have severe pancytopenia. There were no abnormal physical findings except pallor and anemic conjunctiva. The relevant hematological parameters were as follows: red blood cells (RBC), $1.32 \times 10^{6} / \mu \mathrm{l}$; hemoglobin $(\mathrm{Hb}), 5.0 \mathrm{~g} / \mathrm{dl}$; mean corpuscular volume (MCV) $105 \mathrm{fl}$; white blood cells (WBC) 1,900/ $\mu \mathrm{l}$; platelets, $2.4 \times 10^{4} / \mu \mathrm{l}$; and serum erythropoietin $3,030 \mathrm{mU} / \mathrm{ml}$. Other laboratory data were as follows: serum Fe, $205 \mu \mathrm{g} / \mathrm{dl}$; TIBC, $183 \mu \mathrm{g} / \mathrm{dl}$; vitamin $\mathrm{B}_{12}, 870 \mathrm{pg} / \mathrm{ml}$; normal NAP (score 408, positive rate $98 \%$ ), negative sugar water and HAM test. Other chemistry, including lactate dehydrogenase (LDH), and coagulation data were within normal limits. Bone marrow smears and biopsy specimens (Fig. 1, left) revealed marked hypocellularity with subtle dyserythropoiesis; i.e. megaloblastoid maturation or multinuclearity, but without

From the First Department of Internal Medicine, Hirosaki University School of Medicine, Hirosaki

Received for publication August 6, 1997; Accepted for publication November 28, 1997

Reprint requests should be addressed to Dr. Yoshiko Tamai, the First Department of Internal Medicine, Hirosaki University School of Medicine, 5 Zaifu-cho, Hirosaki 036-8216 


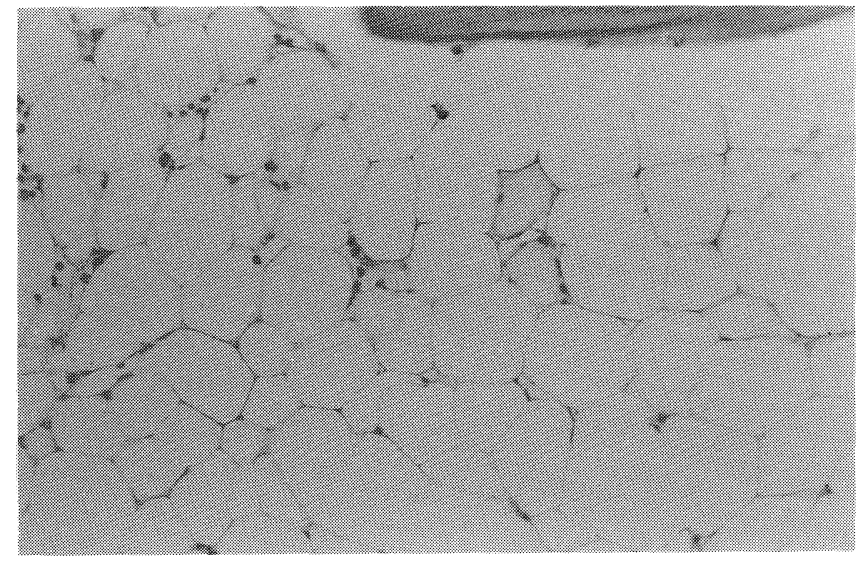

A

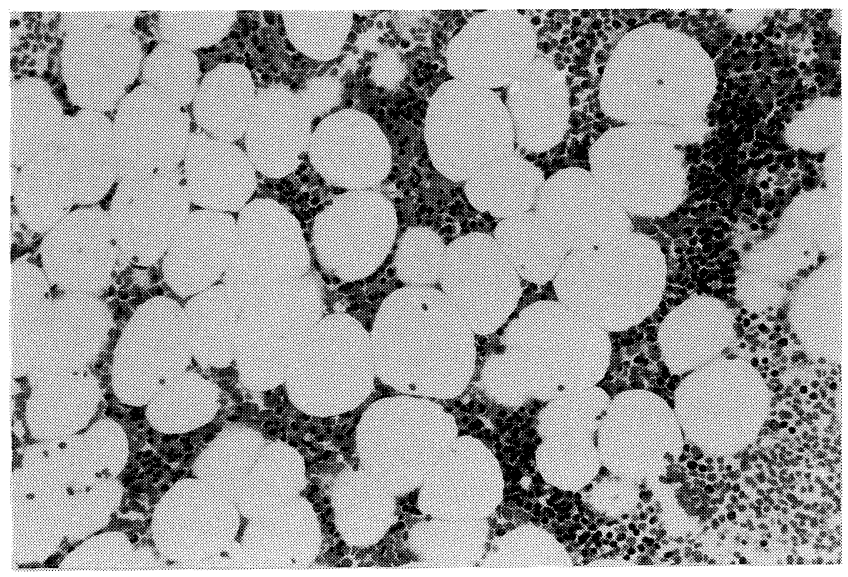

$\mathrm{B}$

Figure 1. (A) Bone marrow specimen at diagnosis (January 1991), (May-Giemsa stain, $\times$ 200). (B) Bone marrow specimen (March 1997), (May-Giemsa stain, $\times 200)$.

dysmegakaryopoiesis and abnormalities of granulocytes (pseudo-Pelger cell or agranular granulocyte) and no increase in blast cells. Bone marrow scintigraphy indicated reduced bone marrow hematopoiesis. At that time the patient had normal karyotype and was diagnosed with a moderate aplastic anemia.

At first, he was treated with a combination of androgen (metenolone acetate, $30 \mathrm{mg} /$ day) and glucocorticoid (prednisolone, $30 \mathrm{mg} /$ day) based on the therapies of aplastic anemia, but did not respond to treatment and was subsequently transfused with 6 to 8 units of concentrated red cells per month.

Bone marrow aspiration and chromosomal examination were repeated in July 1991, because the patient had severe macrocytic anemia and occasional erythroblasts in the peripheral blood. The bone marrow specimen still revealed hypocellularity with mild dyserythropoiesis, but without an increase in ringed-sideroblasts. Karyotypic analysis of the bone marrow showed 47,XY,+8/46,XY,+8, -19/47,XY,+Y/ $47, X Y,+\operatorname{mar}(14 / 20$ cells). It appears that the aplastic anemia progressed to hypoplastic myelodysplastic syndrome (refrac- tory anemia; RA), although the blast cells were not increased. From February 1992 to October 1993, he was treated with vitamin $\mathrm{D}_{3}(3 \mu \mathrm{g} / \mathrm{day})$, and despite administration for more than 1 year, his hematopoiesis did not recover.

The rhEPO (epoetin beta) was administrated from May 1992. At that time his serum EPO was $5,260 \mathrm{mU} / \mathrm{ml}$, prior to treatment with $6,000 \mathrm{U}$ of rhEPO 3 times a week. Over a treatment course of 4 weeks the patient's hemoglobin concentration gradually increased and consequently his requirement for concentrated red cell transfusions decreased more than $50 \%$. No transfusions were required after rhEPO was increased to $12,000 \mathrm{U}, 3$ times a week, beginning in January 1993. Since the beginning of treatment the patient received a total of $108 \mathrm{U}$ of concentrated red cells, 94 of which were given before initiation of rhEPO therapy. The dosage of rhEPO was decreased to 12,000 U, 2 times a week, beginning in April 1995, and further reduced to $24,000 \mathrm{U}$ once a week beginning in March 1996.

Bone marrow aspirates, obtained in March 1997, still showed hypoplasia, but with areas of focal hyperplasia, which was regarded as an erythroid reaction (Fig. 1, right). The patient still has chromosomal abnormalities of $47, \mathrm{XY},+8(13 / 20)$, but other previously detected abnormalities were not apparent. The patient shows no evidence of progression to high risk MDS or acute leukemia, and has maintained a good response to rhEPO for more than 60 months without any adverse side effects. His most recent peripheral blood and blood chemistry data in March 1997 are as follows: RBC, $3.85 \times 10^{6} / \mu \mathrm{l} ; \mathrm{Hb}, 14.3 \mathrm{~g} / \mathrm{dl} ; \mathrm{MCV}$

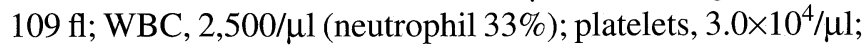
serum Fe $44 \mu \mathrm{g} / \mathrm{dl}$; TIBC $179 \mu \mathrm{g} / \mathrm{dl}$; and serum EPO, $107 \mathrm{mU} /$ $\mathrm{ml}$. A summary of the patient's clinical course is shown in Fig. 2.

\section{Discussion}

In this paper, we report a patient with a hypoplastic MDS which showed excellent response to rhEPO of more than 5-year duration. He had been treated with androgen and glucocorticoid, but there was no response. Although it is undeniable that vitamin $D_{3}$ was effective in part for erythropoiesis, the decreasing erythropoiesis was not seen after vitamin $D_{3}$ was stopped; therefore it seems to have little effect on the hematopoiesis in this case.

Treatment of MDS-associated anemia with rhEPO has been widely studied $(3,4)$. The response rate was $24 \%$ by the combined analysis of a published trial with 423 MDS patients (1). The association of responsiveness to EPO treatment with a low serum EPO level $(3,5)$, low risk subtype of the FAB criteria (6), non-transfused (3) or newly diagnosed (6), normal LDH (6), normal cytogenetics $(5,6)$ and absolute reticulocyte counts (5) has been shown. However, some reports (6-8) showed that there is no correlation between the pre-treatment serum EPO level or observed/predicted EPO ratio and rhEPO response. Yoshida et al (8) showed a progressive decline in serum EPO levels in six of seven responders, even while on rhEPO therapy, and proposed monitoring serum EPO levels during treatment 


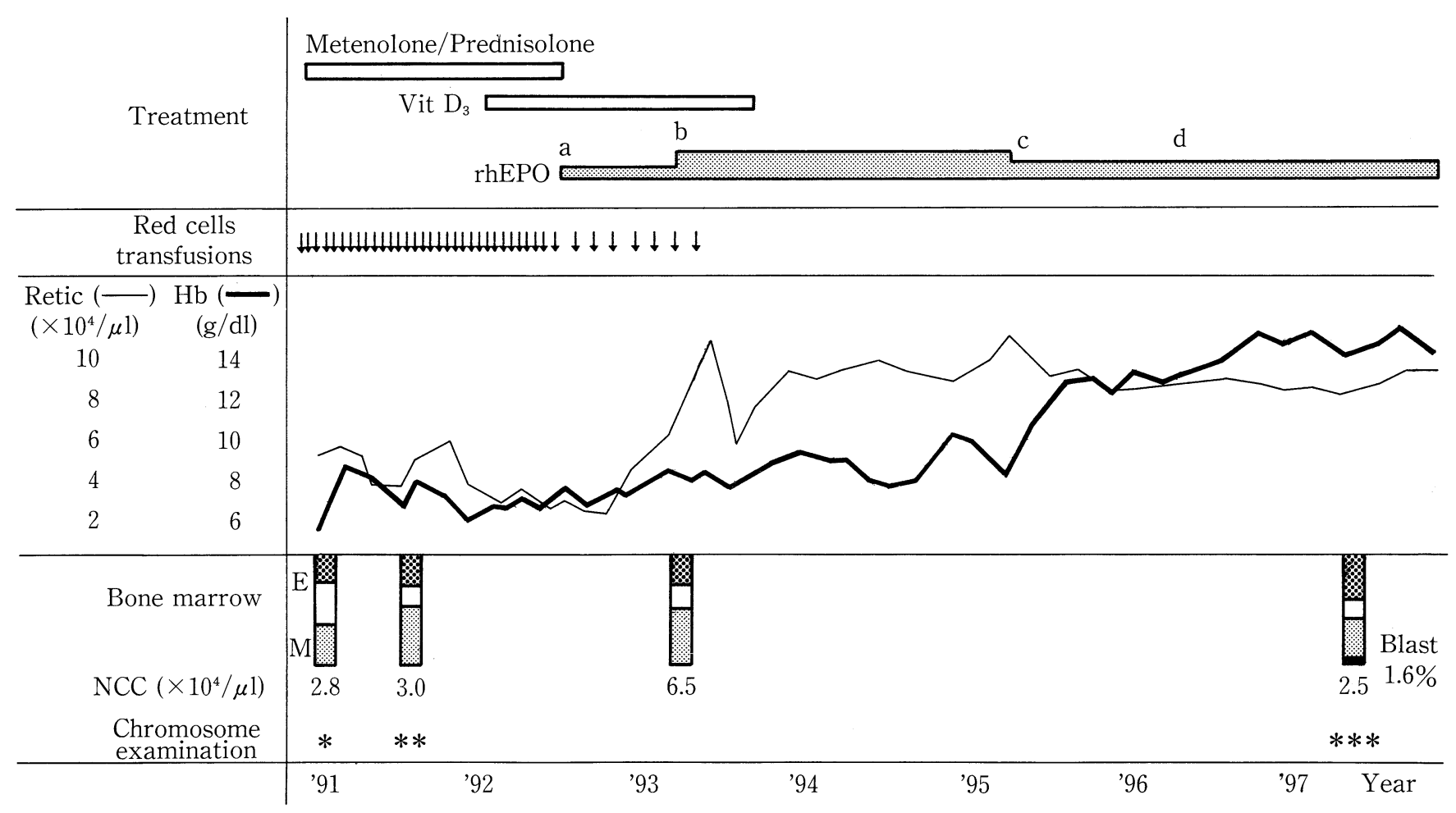

Figure 2. Clinical course of the patient. The patient required about 8 units of red cells per month (indicated by arrows). After starting rhEPO therapy, a marked increase in reticulocytes was observed with a subsequent decrease in transfusions. The patient has not received any transfusions since March 1993. Metenolone and prednisolone were administrated orally at $30 \mathrm{mg} /$ day, and activated vitamin $D_{3}$ was given at $3 \mu \mathrm{g} /$ day. Doses of rhEPO were as follows: 6,000 $\mathrm{U} 3$ times a week from May 1992 (a); 12,000 U 3 times a week, beginning in January 1993 (b); 12,000 U 2 times a week, from April 1995 (c); and 24,000 U once a week from March 1996 (d). Bone marrow aspirates were examined at indicated time points. Chromosomal examinations were performed at times indicated by the asterisks: *: 46,XY, **: 47,XY,+8 (10/20)/ $46, X Y,+8,-19(1 / 20) / 47, X Y,+Y(2 / 20) / 47, X Y,+$ mar $(1 / 20)$ and 6 cells showed 46,XY, ***: 47,XY,+8 (13/20) and another 46,XY. Throughout the entire clinical course the patient's WBC and platelet counts did not change significantly (data not shown). E: erythroid portion (\%), M: myeloid portion (\%), NCC: nucleated cell counts $\left(\times 10^{4} / \mu \mathrm{l}\right)$, Retic: reticulocyte $\left(\times 10^{4} /\right.$ $\mu \mathrm{l})$, rhEPO: recombinant human erythropoietin (epoetin beta), Vit $D_{3}$ : vitamin $D_{3}$ (alfacalcidol).

with rhEPO. The serum EPO levels in the present case also showed a marked decline, indicating a good response to rhEPO, in agreement with the data of Yoshida et al (8).

Our hypoplastic MDS patient has been free from blood transfusions or any symptoms of anemia for more than 60 months. Because of the following reasons, it is likely that he has maintained a good response to rhEPO, rather than the result of the natural course of the disease: 1) The patient's white blood cell and platelet counts did not change throughout the entire clinical course, 2) in this case, there seems to be a correlation between the dose of administration of rhEPO and the response of erythropoiesis.

It is very important to identify good-responders to rhEPO, since rhEPO therapy for patients with MDS have a high cost/ benefit ratio because of the high drug cost and the low response rate (less than one-third of treated patients respond). Compared to concentrated red cell transfusions, rhEPO therapy is an expensive treatment, even in patients that respond, however, rhEPO therapy alleviates many complications of blood transfu- sions. Presently, we do not have firm criteria to determine which MDS patients should be treated with rhEPO. Di Raimondo et al (6) showed a good response rate $(9 / 12)$ to rhEPO alone by selecting the MDS patients. The present patient had an excellent response to rhEPO therapy despite a very high serum EPO level, multiple transfusions, severe thrombocytopenia and chromosomal abnormalities.

In this patient, the abnormal karyotype $(47, \mathrm{XY},+8)$ still remained and the proportion of this clone was not changed in March 1997. The presence of this abnormality after the improvement of erythropoiesis suggests that both normal and abnormal hematopoietic clones may be stimulated to proliferate by rhEPO.

An unanswered question regarding rhEPO treatment concerns the duration of the therapy; there have been few studies of the duration of the positive response. The clinical utility of rhEPO treatment for MDS depends on the response duration as well as the response rate. Negrin et al (5) reported that the median duration was 11 months in 17 patients with erythroid 
responses to rhEPO alone or with granulocyte colony-stimulating factor (G-CSF), with 6 patients having relatively prolonged and durable responses for 15 to 36 months. One of the reasons for the loss of response to EPO may be EPO resistance. Mittelman (4) described that EPO resistance is similar to insulin resistance in that more EPO than initially required is needed to achieve the same effect. It is worthy of special mention that the present patient has maintained a good response to rhEPO for more than 60 months and works as a farmer without symptoms. Furthermore, it was possible to decrease the rhEPO dose and maintain a good response.

The present case suggests that the anemia in some patients with MDS can improved with long-term, low doses of rhEPO (400 to $800 \mathrm{U} / \mathrm{kg} /$ week), even though the patient may be predicted to be a poor-responder to rhEPO because of markedly high pre-treatment serum EPO levels. Since there are few adverse effects during rhEPO therapy, it is worth trying once for low-risk MDS patients depending on concentrated red cell transfusion requirements.

\section{References}

1) Ganser A, Hoelzer D. Clinical use of hematopoietic growth factors in the myelodysplastic syndromes. Semin in Hematol 33: 186, 1996.

2) Yoshida Y. Treatment of the myelodysplastic syndromes: An updated Japanese experience. Semin in Hematol 33: 246, 1996.

3) Hellström-Lindberg E. Efficacy of erythropoietin in the myelodysplastic syndromes: a mete-analysis of 205 patients from 17 studies. Br J Haematol 89: 67, 1995.

4) Mittelman M. Recombinanterythropoietin in myelodysplastic syndromes: Whom to treat and how? More questions than answers. Acta Haematol 90: 53, 1993.

5) Negrin RS, Stein R, Doherty K, et al. Maintenance treatment of the anemia of myelodysplastic syndromes with recombinant human granulocyte colony-stimulating factor and erythropoietin: Evidence for in vivo synergy. Blood 87: 4076, 1996.

6) Di Raimondo F, Longo G, Cacciola Jr E, et al. A good response rate to recombinant erythropoietin alone may be expected in selected myelodysplastic patients. A preliminary clinical study. Eur J Haematol 56: 7, 1996.

7) Bucalossi A, Marotta G, Bigazzi C, et al. Use of a functional classification of anemia in myelodysplastic syndromes in identify subgroups of patients responsive to recombinant human-erythropoietin therapy. Eur J Haematol 56: 106, 1996.

8) Yoshida Y, Anzai N, Kawabata H, Kohsaka Y, Okuma M. Serial changes in endogenous erythropoietin levels in patients with myelodysplastic syndromes and aplastic anemia undergoing erythropoietin treatment. Ann Hematol 66: 175, 1993. 